\title{
Experiencias escolares de jóvenes retornados de EUA a secundarias fronterizas de México
}

\author{
Porfiria del Rosario Bustamante De la Cruz, Mg. \\ Profesora en la Universidad Autónoma de Baja California, \\ México*
}

pbustamante@uabc.edu.mx

\section{Resumen (analítico)}

En este artículo se explora una serie de experiencias escolares que viven las y los jóvenes de secundaria que, después de haber estado por un tiempo significativo en escuelas estadounidenses, han retornado y ahora cursan estudios en instituciones mexicanas. La metodología utilizada fue cualitativa; se empleó un cuestionario sociodemográfico y se tomó como punto de partida la etnografía escolar. La población estuvo conformada por veinte estudiantes retornados de cinco secundarias públicas de Mexicali, Baja California, a quienes se les aplicó el cuestionario. Asimismo, se realizaron nueve entrevistas en profundidad. Los principales resultados obtenidos señalan la clasificación de las experiencias escolares identificadas por los propios estudiantes retornados, divididas en infraestructura de la escuela, discontinuidad del sistema educativo y cultura escolar.

\section{Palabras clave}

México, migración de retorno, jóvenes, estudiantes extranjeros, diversidad cultural, conflictos culturales, proceso de interacción educativa, enseñanza secundaria.

\section{Thesauro}

Tesauro de Ciencias Sociales de la Unesco.

\section{Para citar este artículo}

Bustamante de la Cruz, P. del R. (2020). Experiencias escolares de jóvenes retornados de EUA a secundarias fronterizas de México. Revista Latinoamericana de Ciencias Sociales, Niñez y Juventud, 18(2), 1-20. http://dx.doi.org/ 10.11600/1692715x.18201

\section{Historial}

Recibido: 29.04.2019

Aceptado: 08.07.2019

Publicado: 17.03.2020

\section{Información artículo}

Este artículo hace parte del proyecto «Estudiar en tiempos de muros: narrativas de jóvenes (in)migrantes de México y EUA en secundarias de Mexicali, B. C. y en high school de Flagstaff, AZ». También retoma algunas experiencias escolares de las y los jóvenes retornados que surgieron en el trabajo «Disonancias culturales entre alumnos retornados de EUA a Mexicali, Baja California» (Bustamante, 2016) realizado entre agosto de 2014 y julio de 2016. Ambos fueron financiados por el Consejo Nacional de Ciencia y Tecnología del Gobierno de México. Área de conocimiento: Otras ciencias sociales; subárea: Educación general. 


\section{Experiences of young people who return from the USA in secondary schools near the Mexican border}

Abstract (analytical)

This paper describes the secondary school experiences of Mexican young people after having spent a significant period of time attending American schools. The methodology used in the study was qualitative, supported by a socio-demographic survey inspired based on an ethnography of the schools. The population for the study consisted of 20 returned students studying in five public schools in Mexicali, Baja California, who responded to the questionnaire; besides. Nine in-depth interviews of these students were conducted. The responses of the returned students highlighted differences in school infrastructure, discontinuity in the educational system and school culture.

\section{Keywords}

Mexico, returning migration, youth people, foreign students, cultural diversity, cultural conflicts, educational interaction process, middle school.

\section{Experiências escolares de jovens retornados dos EUA para escolas secundárias no México}

\section{Resumo (analítico)}

Neste artigo exploram-se as experiências escolares de jovens mexicanos de ensino médio retornantes ao sistema de ensino mexicano após longos períodos de imigração nos Estados Unidos. A metodologia utilizada é qualitativa, apoiada num questionário sociodemográfico e inspirada na etnografia escolar, enquanto a população do estudo consistiu de 20 estudantes retornantes localizados em cinco escolas públicas de ensino médio em Mexicali, Baja California, com quem o questionário foi trabalhado. Além disso foram aplicadas nove entrevistas em profundidade. O principal resultado obtido é a classificação das experiências escolares identificadas pelos proprios estudantes retornantes, dividindo-se na infraestrutura da escola, a discontinuidade do sistema educacional e na cultura escolar.

\section{Palavras-chave}

México, retornar migração, jovens, estudantes extrangeiros, diversidade cultural, conflito cultural, processo de interação educacional, ensino secundário.

Información sobre la autora

[*] Profesora de asignatura y maestra en Estudios Socioculturales en la Universidad Autónoma de Baja California. Doctoranda del Instituto de Investigaciones en Educación de la Universidad Veracruzana (México). Índice H5: 6. (iD) 0000-0001-6562-9609. Correo electrónico: pbustamante@uabc.edu.mx 


\section{Introducción}

En los últimos años se ha presentado una creciente migración de retorno, nunca antes presenciada, desde los Estados Unidos de América (EUA) a México. En este fenómeno se reconocen como protagonistas a niñas, niños y jóvenes (NNJ) en edad escolar obligatoria. En México, la migración de retorno - al darse en formas y escenarios diferentesafecta a las escuelas, lo que representa un reto para la cultura escolar. Esta situación pone en escena a las y los llamados jóvenes retornados, quienes, después de estudiar por un tiempo considerable en el sistema educativo estadounidense, se encuentran en escuelas mexicanas. De acuerdo con Valdez y García (2017), las y los estudiantes retornados son aquellos nacidos en México y en EUA que regresan a México de forma voluntaria o involuntaria. Si bien las y los jóvenes nacidos en EUA se pueden clasificar como mexicoamericanos o binacionales, en este trabajo se consideran como retornados por la nacionalidad mexicana de uno o de los dos padres. Este hecho produce un sentido de pertenencia respecto a México y causa un retorno significativo.

Una vez inscritos en escuelas mexicanas, las y los jóvenes retornados pasan por experiencias culturales conflictivas, tanto en su ingreso escolar como en las interacciones con la comunidad educativa (compañeros, docentes, directores, etc.). Esta población tiene referentes y códigos culturales de la escuela estadounidense que, de acuerdo con las costumbres y tradiciones establecidas en México, permean la cultura escolar e incluso van más allá de esta. Ante tal panorama, es importante abordar las experiencias de dichos actores para conocer la realidad que viven tras el ingreso a su nueva escuela.

Vista de este modo, la vida en las escuelas mexicanas trasciende la práctica educativa. Existe una dimensión sociocultural que puede ser clave para el éxito o la deserción escolar de las y los jóvenes retornados. Actualmente, el mundo es cada vez más diverso; de ahí que se identifique, acepte y trabaje con la diversidad en las escuelas. Para conocer las experiencias escolares de esta población es indispensable pensar desde otros enfoques que permitan vislumbrar la forma en que interactúa toda la comunidad educativa. Esto 
es algo que reconfigura las prácticas y actitudes de todas y todos los actores sociales de las instituciones. De acuerdo con Rockwell (1995), las experiencias escolares contemplan las pautas, relaciones y costumbres que caracterizan la escuela, las cuales se proyectan hacia el entorno social inmediato y amplían, así, el ámbito formativo de la institución hacia los contextos familiar, laboral y de la vida civil. A propósito de esta idea, se considera que las y los jóvenes retornados construyen sus propias experiencias mediante una interacción en el entorno escolar que, a su vez, es moldeado por el contexto general.

Respecto a los lugares de arribo de las y los jóvenes retornados a México, Aguilar y Ortiz (2018) plantean que esta población se concentra en diez estados; en orden de importancia estos son: Baja California, Chihuahua, Jalisco, Tamaulipas, Michoacán, Sonora, Guanajuato, Estado de México, Puebla y Coahuila.

Entre las dificultades que atraviesa esta población se encuentra la de que es invisibilizada, dada la poca o nula evidencia de su origen educativo o de su movilidad en uno u otro país, así como el sentimiento de frustración y no reconocimiento en el aula. Esta segunda cuestión se relaciona con el incumplimiento de tareas porque su realización implica leer, escribir y, por ende, comprender contenidos en español.

Es así como, según la Dirección General de Relaciones Internacionales (DGRI, 2015), las y los estudiantes retornados piensan que en México los maestros no responden a sus necesidades debido a la diferencia del idioma y de la cultura. Esta población considera que el sistema educativo de EUA les otorga más actividades extras para desarrollar su potencial y se frustran al enfrentarse a cambios que consideran drásticos, relacionados con el contenido curricular, el conjunto de materias y el diseño de actividades. Por su parte, Vargas (2019) sostiene que las barreras educativas que enfrenta la población juvenil de retornados se pueden clasificar en estructurales, culturales y sociales. Las primeras se relacionan con el acceso a la escuela (forman parte de ellas la matriculación y la disponibilidad de cupo, así como los documentos); las segundas son dificultades derivadas del manejo del español y de las diferencias socioculturales en la escuela; las últimas tienen que ver con la separación familiar y la falta de redes sociales en la escuela.

No obstante, esta situación no es nueva. Una de las iniciativas para atender a estudiantes migrantes en edad escolar obligatoria se puso en práctica en 1976 en los estados de Michoacán y California, actuando en colaboración los sistemas educativos de México y EUA. Sus resultados dieron origen al Programa Binacional de Educación Migrante (Probem) en 1982. Posteriormente, hacia 1990, en Washington, D. C., se firmó el Memorándum de entendimiento sobre educación entre los Gobiernos de México y EUA (Instituto de los 
Mexicanos en el Exterior [IME], 2016). Este programa intenta abordar las necesidades de índole pedagógica e inclusiva de la población infantojuvenil de retornados. No obstante, cada entidad federativa trabaja de forma diferente. En el caso de Mexicali, Probem trabaja mediante talleres de apoyo a jóvenes «extranjeros» del nivel de secundaria.

Las estadísticas de Probem (2018), proporcionadas por la Coordinación de Mexicali, muestran un aumento significativo de jóvenes que retornan. En el ciclo escolar 2015-2016 hubo un registro de 2859 estudiantes de secundaria; pero las estadísticas siguen aumentando: entre 2016 y 2017 ascendió a 3039; entre 2017 y 2018, a 3323; entre 2018 y 2019, a 3527; y en el ciclo escolar 2019-2020, a 3532 estudiantes. Este aumento pone en evidencia la importancia del tema y la necesidad de conocer las realidades que viven las jóvenes y los jóvenes retornados. Es necesario considerar esta investigación como un precedente ante el reordenamiento social actual tras la toma de posesión de Donald Trump como presidente de EUA (a principios de 2017) y de Andrés Manuel López Obrador, elegido primer mandatario de México a finales de 2018.

Hay que reconocer el retorno como un proceso no lineal, familiar, heterogéneo y complejo en el que migrar de «regreso» a México desde EUA es una oportunidad para la familia (Valdez \& Ruiz, 2017). Así pues, en este trabajo se analizan las experiencias escolares de la población juvenil de retornados de secundaria en Mexicali (Baja California) durante el ciclo escolar 2015-2016. El trabajo aborda el tema haciendo énfasis en la dimensión sociocultural y en el desarrollo cultural desde el área pedagógica-educativa, y reafirma, de acuerdo con Zúñiga (2017), la urgencia de poner en escena a los NNJ migrantes; en especial las dificultades que viven en el momento de incorporarse a la escuela.

\section{Método}

La metodología propuesta para este estudio fue de tipo cualitativo y se basó en un enfoque fenomenológico que permitiera analizar las representaciones de las y los jóvenes retornados inscritos en escuelas secundarias de Mexicali. Este estudio es transdisciplinar, al tomar en cuenta campos como la pedagogía, la sociología y la antropología. De igual modo, se desarrolló desde una perspectiva sociocultural, entendida como la atención prestada a las formas de relación y de organización en las que tiene lugar cierto comportamiento mediado por determinados sentidos y significados. 
El estudio contó con tres ejes en el trabajo de campo: el primero implicó la identificación de las y los jóvenes retornados en las secundarias; posteriormente, se aplicaron los cuestionarios, de los que se desprendieron los códigos que apoyaron la selección de la escuela para realizar la etnografía, así como la selección de las y los nueve actores con los que se trabajó la entrevista a profundidad. Está se constituyó en el tercer eje.

Para llevar a cabo esta investigación se seleccionó una muestra de veinte jóvenes retornados inscritos en cinco colegios de secundaria (cuatro estatales y uno federal), cuyas edades variaban entre los doce y los dieciséis años. El proceso de selección de la muestra fue intencional y se basó en los datos proporcionados por Probem en 2016, durante su primera intervención en la ciudad. Para obtener la información se aplicó un cuestionario (Blanco, 2011) que incluía cinco áreas: 1) datos generales (sexo y edad, origen, residencia actual y nivel socioeconómico); 2) aspectos familiares (número de personas con las que vive y origen de los padres y hermanos); 3 ) historia migratoria escolar (país de nacimiento, de inicio de sus estudios, motivos de su retorno a México y periodos de residencia en EUA); 4) contexto sociocultural en ambos países, y 5 ) aspectos educativos (actividades).

La tabla 1 expone algunos datos obtenidos de los veinte cuestionarios, como la escuela a la que los estudiantes asistían, sexo, nacionalidad, edad, grado escolar, trayectoria entre México y EUA (lugar de origen y lugar destino) y el último año de arribo a Mexicali.

A partir de los veinte cuestionarios aplicados se realizaron nueve entrevistas en profundidad (Taylor \& Bogdan, 1990) a estudiantes retornados que colaboraron de forma voluntaria. Se seleccionaron a las cuatro jóvenes de la escuela dos y a las y los jóvenes de la escuela cinco. Como requisito para las entrevistas se emplearon dos consentimientos informados: uno dirigido al padre de familia o tutor de las y los jóvenes, y el segundo remitido a estos últimos. También se emplearon los principios de la etnografía educativa (Rockwell, 2015) como apoyo para las entrevistas en profundidad. Así, se pudo describir, explicar e interpretar la cultura de la escuela y lo que rodea a los estudiantes en una institución de educación básica de Mexicali. La etnografía se realizó en la secundaria cinco y se tomaron en cuenta dos criterios: el primero - considerado el de mayor relevanciaes que en dicha escuela había cinco jóvenes retornados y tres de ellos asistían al mismo salón; el segundo fue el interés mostrado por las autoridades de la secundaria.

El ejercicio etnográfico tuvo una duración de un año. Durante los primeros seis meses se desarrolló el acercamiento a la población en cuatro momentos: 1) con Probem; 2) en los colegios de secundaria a los que asisten jóvenes; 3 ) al solicitar el permiso a las autoridades educativas; y 4) al contactar cara a cara a los jóvenes. El resto del año se trabajó directamente con 
estos actores y con sus prácticas culturales y cotidianidades escolares, tanto en el aula de clases como en los espacios recreativos, deportivos y de ocio, de modo que la observación participante y no participante, junto con el diario de campo (Velasco \& Díaz de Rada, 1997), fueron esenciales para la recolección de la información; además se construyó una empatía con las y los jóvenes, la cual permitió conocer la realidad a la que están sujetos.

\section{Tabla 1}

Estudiantes retornados inscritos en secundarias públicas de Mexicali (Baja California)

\begin{tabular}{|c|c|c|c|c|c|c|}
\hline $\begin{array}{l}\text { Escuela } \\
\text { secundaria }\end{array}$ & Sexo & Nacionalidad & $\begin{array}{l}\text { Edad en } \\
\text { años }\end{array}$ & $\begin{array}{l}\text { Grado } \\
\text { escolar }\end{array}$ & $\begin{array}{c}\text { Trayectoria migratoria entre } \\
\text { México y EUA }\end{array}$ & $\begin{array}{l}\text { Último año } \\
\text { de arribo }\end{array}$ \\
\hline \multirow{3}{*}{1} & M & Estadounidense & 15 & $3^{\circ} \mathrm{A}$ & EUA-Mxli/Mxli-EUA/EUA-Mxli & 2015 \\
\hline & M & Estadounidense & 14 & $3^{\circ} \mathrm{A}$ & EUA-Mxli & 2014 \\
\hline & M & Mexicana & 13 & $2^{\circ} \mathrm{A}$ & Mxli-EUA/EUA-Mxli & 2015 \\
\hline \multirow{4}{*}{2} & M & Estadounidense & 14 & $3^{\circ} \mathrm{C}$ & $\begin{array}{l}\text { EUA-Gto/ Gto-EUA/EUA-Mxli/ } \\
\text { Mxli-EUA/EUA-Mxli/Mxli-EUA/ } \\
\text { EUA-Mxli/ }\end{array}$ & 2014 \\
\hline & M & Estadounidense & 13 & $2^{\circ} \mathrm{E}$ & $\begin{array}{l}\text { EUA-Mxli/Mxli-EUA/EUA-Mxli/ } \\
\text { Mxli-EUA/EUA-Mxli/ }\end{array}$ & 2015 \\
\hline & M & Estadounidense & 13 & $2^{\circ} \mathrm{E}$ & EUA-Mxli/Mxli-EUA/EUA-Mxli & 2015 \\
\hline & $\mathrm{H}$ & Estadounidense & 12 & $1^{\circ} \mathrm{D}$ & EUA-Mxli & 2010 \\
\hline \multirow{4}{*}{3} & $\mathrm{H}$ & Estadounidense & 15 & $3^{\circ} \mathrm{A}$ & EUA-Son (Son-Mxli) & 2011 \\
\hline & $\mathrm{H}$ & Estadounidense & 13 & $2^{\circ} \mathrm{C}$ & EUA-Mxli/Mxli-EUA/EUA-Mxli & 2010 \\
\hline & M & Estadounidense & 14 & $3^{\circ} \mathrm{D}$ & EUA-Mxli/Mxli-EUA/EUA-Mxli & 2010 \\
\hline & M & Estadounidense & 13 & $2^{\circ} \mathrm{A}$ & EUA-Mxli/Mxli-EUA/EUA-Mxli & 2013 \\
\hline \multirow{4}{*}{4} & M & Estadounidense & 15 & $2^{\circ} \mathrm{C}$ & EUA-Mxli & 2011 \\
\hline & $\mathrm{H}$ & Estadounidense & 15 & $2^{\circ} \mathrm{D}$ & EUA-Mxli & 2007 \\
\hline & $\mathrm{H}$ & Estadounidense & 15 & $3^{\circ} 1$ & EUA-Mxli & 2008 \\
\hline & M & Estadounidense & 15 & $3^{\circ} \mathrm{C}$ & EUA-Mxli/Mxli-EUA/ EUA-Mxli & 2011 \\
\hline \multirow{5}{*}{5} & M & Mexicana & 15 & $1^{\circ} \mathrm{E}$ & $\begin{array}{l}\text { Mxli-EUA/EUA-Mxli/ Mxli- } \\
\text { EUA/EUA-Mxli }\end{array}$ & 2014 \\
\hline & M & Mexicana & 15 & $3^{\circ} \mathrm{C}$ & Mxli-EUA/EUA-Mxli & 2014 \\
\hline & M & Estadounidense & 15 & $3^{\circ} \mathrm{E}$ & EUA-Mxli/Mxli-EUA/EUA-Mxli & 2009 \\
\hline & M & Estadounidense & 15 & $3^{\circ} \mathrm{C}$ & EUA-Mxli & 2012 \\
\hline & $\mathrm{H}$ & Estadounidense & 16 & $3^{\circ} \mathrm{C}$ & EUA-Mxli & 2012 \\
\hline
\end{tabular}

Nota. ${ }^{a}$ Mexicali: Mxli; Sonora: Son; Guanajuato: Gto. 
El sistema de categorización aplicado en el trabajo fue libre y se usó Atlas.ti (versión 8), herramienta que posibilitó la codificación de los datos mediante las bases de la teoría fundamentada (Glaser, 1992). En el análisis de las entrevistas sobre el proceso que vivió la población juvenil de retornados al incorporarse a escuelas de secundaria del sistema educativo mexicano se tomó en cuenta la saturación de datos que emergían en torno a la frecuencia. Así, del análisis surgieron temas que permitieron comprender las realidades a las que están sujetos los actores.

Para analizar los datos obtenidos en el cuestionario se utilizó el programa Statistical Package for Social Sciences (SPSS), que posibilitó conocer el perfil sociodemográfico de la población juvenil de retornados. Los datos que se utilizaron para generar este perfil consistieron en una genealogía de las experiencias escolares y extraescolares, obtenida de investigaciones que se han realizado en México. Algunas experiencias escolares que sobresalen en el cuestionario tienen que ver con las formas en que los estudiantes retornados son invisibilizados en la escuela, la frustración y falta de reconocimiento, así como la ausencia de atención por parte del docente (DGRI, 2015).

La categoría emergente del análisis de esta investigación son las disonancias culturales, con tres componentes: limitaciones de espacio educativo (Romo, 2012), discontinuidad escolar/sistema educativo (Terigi, 2010) y cultura escolar (Deal \& Peterson, 2009).

\section{Resultados}

Además de ser el lugar donde se da el proceso de enseñanza-aprendizaje, las escuelas son el espacio donde las y los estudiantes pasan la mayor parte del día, después de su hogar. Por tanto, es necesario estudiar lo que sucede en las escuelas donde estos crecen y aprenden de su entorno. Las experiencias escolares que viven las y los jóvenes retornados desde que ingresan al sistema educativo mexicano están atravesadas por disonancias socioculturales; esto es, los conflictos entre los referentes culturales que la población juvenil posee de la escuela estadounidense y los códigos culturales del nuevo contexto escolar (Bustamante, 2016).

En el perfil sociodemográfico de la población juvenil de retornados se destacaron cuatro características: primero, la mayoría de las y los actores son de nacionalidad estadounidense; sin embargo, esta población resignifica el retorno debido al sentido de pertenencia que, respecto a la nacionalidad mexicana, tiene al menos uno de sus padres. 
Segundo, a pesar de que algunos viven un retorno involuntario a México, a trece de las y los estudiantes les gusta vivir en Mexicali por la reunificación familiar y por «la seguridad de estar en su país». Tercero, perciben que la educación adolece de carencias en infraestructura y que la comunidad escolar no tiene información sobre la situación de los alumnos migrantes. Y cuarto, perciben falta de inclusión socioeducativa: independientemente de los años que tengan en el país, se sienten excluidos e incomprendidos.

Para obtener los resultados esperados de la investigación se unificaron los datos del cuestionario, del apoyo etnográfico y de las entrevistas en profundidad. Así, se reconocieron tres áreas en las que las y los jóvenes retornados vislumbraron disonancias culturales. En primer lugar, se encuentran las relacionadas con las limitaciones del espacio educativo en México. Se entiende como espacio educativo el ambiente que incluye los aspectos «físicos, sociales y humanos que configuran el espacio-tiempo [...] en que [el] ser humano vivencia experiencias diversas que le permiten con más o menos facilidad generar apren-dizajes que favorecen su desarrollo integral» (Romo, 2012, p. 143). En este trabajo, el espacio educativo se relaciona con el diseño y el reducido espacio de las escuelas, así como con los espacios de escolarización y recreación: salones de clases, biblioteca, áreas deportivas y recreativas para el receso escolar, mobiliario en mal estado e, incluso, la escasa asistencia para brindar material educativo. En este contexto, las y los estudiantes retornados se frustraron al sentirse en espacios poco convencionales e inadecuados, comparados con lo que esperaban.

En México, el Instituto Nacional para la Evaluación de la Educación (INEE, 2018) estipula que las escuelas necesitan de diversos recursos para operar: «1) infraestructura física escolar; 2) equipamiento y mobiliario escolar; 3) materiales educativos; y 4) personal» (p. 135). Estos recursos son importantes para que la población estudiantil tenga experiencias positivas en su proceso de escolarización.

Observando las condiciones generales de los colegios de secundaria de Mexicali, la población juvenil de retornados destacó que las escuelas son de menor tamaño que las de los EUA; por tanto, algunas de sus percepciones son semejantes a lo que Goffman (2001) definió como la totalización de las instituciones. En general, los estudiantes calificaron la infraestructura de las escuelas como ineficiente, por no contar con aulas, bibliotecas, laboratorios, cafeterías, comedores, pasillos amplios, casilleros, espacios recreativos y de convivencia adecuados, equipamiento y materiales educativos afines. Tales experiencias escolares demostraron la relación existente entre la adecuación del espacio y el proceso 
de enseñanza-aprendizaje, en vista de que estos actores mostraron poco interés por asistir a la escuela.

En segundo lugar, figuran los conflictos que experimentan las y los jóvenes retornados en el sistema educativo mexicano y que derivan de la discontinuidad escolar. Se entiende por discontinuidad escolar el ausentismo, la interrupción y la disminución del tiempo para aprender. Durante este proceso hay múltiples razones y condiciones que estimulan la deserción educativa (Terigi, 2010). Además, se considera necesario pensar en formas de discontinuidad escolar en un contexto migratorio. En este sentido, el primer inconveniente con el que se enfrentaron las y los jóvenes cuando entraron a estudiar en los colegios de secundaria de Mexicali fue el contenido curricular homogeneizador que establece la Secretaría de Educación Pública. Esta tiene como propósito alinear a la población juvenil de retornados con los valores, costumbres, tradiciones, creencias y modelos de conducta convencionales.

Algunos jóvenes experimentaron confusión en la materia de Formación Cívica y Ética por el contenido enseñado, destinado a fomentar la convivencia entre sus pares. A pesar de que los compañeros de clase afirmaban que es una materia «refácil», los retornados la reprobaron. Algo similar sucede con las materias de Historia y Español, áreas sobre las que manifestaron su impotencia, al sentirse en desventaja respecto de sus compañeros de clase. Las autoridades educativas, en lugar de aprovechar los conocimientos de las y los estudiantes retornados, causan malestar en esta población. Sobre este aspecto, Franco (2017) afirma:

[En México, el currículo] se utiliza para niños que viven diferentes realidades sociales y culturales; además, las condiciones de desigualdad permean en las escuelas, es decir, los marginados lejos de recibir una atención prioritaria reciben una atención precaria que la distancia del logro de los aprendizajes esperados. (p. 714)

En los temas relacionados con los valores patrios, las y los jóvenes retornados desconocían las fechas conmemorativas, como el Día de la Independencia (16 de septiembre) y el 5 de mayo (batalla de Puebla), debido a que en los EUA suelen festejar el 5 de mayo como fecha de la Independencia de México. También mencionaron su desconocimiento del significado de las efemérides y los días festivos estipulados en el calendario escolar mexicano. Un ejemplo significativo es el 2 de noviembre, Día de los Muertos, y las múltiples actividades que se realizan, como la elaboración de altares y ofrendas a los difuntos. 
En esta situación, el sistema educativo mexicano debería abordar — desde la interculturalidad- las necesidades educativas, socioculturales e incluso emocionales de los estudiantes migrantes mestizos, pues cualquier estudiante con otros referentes culturales vive y experimenta con extrañeza el proceso de reconocimiento del centro educativo y de la cultura escolar. Estos cambios pueden afectar el desempeño educativo y determinar que tengan que repetir el grado escolar por diversas razones, como la escasez de cupos en el colegio elegido por los padres o, en el caso de esta generación, la solicitud de realizar el apostillamiento. ${ }^{1}$ Cabe señalar que la mayoría de las y los jóvenes retornados desconocía las engorrosas diligencias que hicieron sus padres para inscribirlos y ubicarlos en el grado escolar correspondiente.

La situación de la población juvenil de retornados también invita a repensar la educación desde la perspectiva de los derechos humanos. En efecto, de acuerdo con la Ley General de Derechos de Niñas, Niños y Adolescentes, en México la Comisión Nacional de Derechos Humanos (CNDH, 2018) reconoce a las personas en edad escolar obligatoria «como sujetos de derechos, con capacidad de defenderlos y exigirlos. También señala al Estado, las familias y la sociedad como responsables de garantizar estos derechos de manera progresiva e integral» (p. 10). En otras palabras, la ley no considera que los NNJ sean un grupo vulnerable, pero, dependiendo de las circunstancias, se pueden encontrar en situación de vulnerabilidad. Además, en el proceso migratorio, a esta población primero se la debe concebir como NNJ y luego como migrante (CNDH, 2018).

Otro aspecto que identificaron algunos de los estudiantes retornados en las entrevistas es el desfase de grados. Antes de asistir a escuelas mexicanas, siete de los nueve estudiantes venían con la idea de continuar el grado escolar en el que se encontraban en los EUA, o bien, de continuar en el siguiente grado. Sin embargo, la mayoría de las veces la escuela les asignó un grado escolar previo. Los responsables de realizar este proceso son el Sistema de Educación y Bienestar Social o los directores de las escuelas; sin embargo, no hay un protocolo establecido. En ocasiones, esta situación desmotiva a las y los jóvenes retornados en su desarrollo educativo, debido a que interpretan el retroceso de grado como deficiencia escolar. Con todo, dos de estos jóvenes retornados tomaron esta situación como una oportunidad para aprender «nuevos» conocimientos relacionados con la cultura escolar receptora.

\footnotetext{
${ }^{1}$ En lo que respecta al apostillamiento, en México, para los trámites de inscripción en el nivel básico, se validaron como documento de identidad las actas de nacimiento extranjeras sin apostillar. El Diario Oficial de la Federación registró este requisito en el 2016 (Jacobo-Suárez, 2017).
} 
Si el sistema educativo es complejo para estudiantes «regulares» en escuelas secundarias, resulta más exigente para la población juvenil de retornados, quienes debe hacer un doble esfuerzo en su desarrollo educativo, etapa de transición social de la juventud en la que se busca, de acuerdo con Nateras (2010), la «legitimización en la constitución identitaria del ser jóvenes versus los mundos adultos» (p. 18). En lo que respecta a las formas de ser jóvenes, estos estudiantes ofrecen resistencia cultural, pues no olvidan cómo era su vida en la escuela estadounidense. Así, ser un estudiante retornado representa, para algunos, una lucha para ser aceptado por los nuevos compañeros de clase.

El tercer y último aspecto que tiene un impacto en la escolaridad y el desempeño educativo de la población juvenil de retornados es la cultura escolar (Deal \& Peterson, 2009). De acuerdo con estos autores, la cultura escolar está compuesta de reglas y tradiciones no escritas, normas y expectativas que lo permean todo: el modo en que la gente actúa, cómo se visten, de qué hablan, si buscan o no ayuda en sus colegas y cómo se sienten los docentes en su trabajo y con sus estudiantes (Deal \& Peterson, 2009). MartínezOtero (2007) explica que, al no estar aislada de su contexto inmediato,

la interdependencia entre cultura y escuela es tan íntima que entre ambas se produce una fusión. La cultura escolar es educativa en el sentido de que cala en la personalidad. Por otro lado, cada miembro de la comunidad contribuye con un sello a generar esa cultura. Es oportuno hacer estos comentarios porque frecuentemente se describe al sujeto como un mero receptor, sin reparar en que cada persona es también constructora de cultura. (pp. 39-40)

Retomando la disonancia de la cultura escolar identificada por las y los jóvenes retornados, los puntos que contiene variaron según sus experiencias escolares. La primera disonancia tiene que ver con las interacciones escolares entre alumnos, es decir, con la proxemia o acercamiento físico, y comprende todas las formas de ser estudiante en la escuela, desde los saludos y despedidas hasta la distancia para hablar y conocerse. Coll (1984) afirma que estas pautas de convivencia constituyen una transgresión decisiva de «aspectos tales como el proceso de socialización, en general, o la adquisición de competencias y de destrezas sociales, [...] el grado de adaptación a las normas establecidas, [...] el nivel de aspiración e incluso el rendimiento escolar» (pp. 119-120).

De igual manera, la convivencia en la escuela y en el aula de los integrantes de la comunidad escolar tiene que ver con relaciones de poder entre las y los docentes, prefectos y directores. La población estudiada mencionó que las autoridades escolares desco- 
nocen sus experiencias como migrantes y aspectos socioculturales que les son propios. Esto se refleja en el nulo apoyo para la inclusión en el salón de clases, donde las y los retornados se sienten invisibles y perciben que sus conocimientos sobre la escuela en los EUA no son valorados. De acuerdo con García y Burgueño (2017), en el proceso de incorporación, la escuela y el aula sitúan a esta población en un contexto en el que «se enfrentan a nuevas formas de asimilar la realidad, pues tienen que asimilar nuevos elementos culturales que tienen que ver desde el uso de un lenguaje (incluso corporal) distinto, hasta nuevos contenidos académicos y formas de relacionarse» (p. 23o).

En efecto, la convivencia trasciende el plano educativo y la subjetividad de la población juvenil de retornados, de modo que las relaciones interpersonales atraviesan el plano del éxito o la deserción escolar. Por otro lado, en la etnografía se identificó que la violencia escolar es una experiencia general entre las y los estudiantes retornados. Las experiencias en el aula varían según la perspectiva de las y los actores retornados. Allí se hace sentir la polisemia del concepto de violencia, que los estudiantes perciben como «carrilla, bullying o cura (falta de respeto de los otros alumnos)». Lo que para algunos estudiantes significa un ritual de inclusión (tal es el caso de la «cura»), para otros representa exclusión o segregación. Este aspecto se relaciona con las interacciones de toda la población estudiantil dentro y fuera de la escuela, donde lo que para unos puede ser una experiencia fortuita, para otros representa lo contrario.

El tercer aspecto por el que pasaron algunos jóvenes retornados es el «acto cívico» (honores a la bandera), considerado por ellos como una actividad innecesaria por su duración (una hora), que a su juicio podría destinarse a clases. Algunos jóvenes retornados coincidieron en que fue una actividad cansina y tediosa, porque el acto obligaba a permanecer de pie y porque tenían que memorizar coros en los que se entonaba el himno nacional y se recitaba el juramento a la bandera. Esta población cuestionó la existencia de un grupo de compañeros de la escuela que conforma la escolta, así como los símbolos patrios, las efemérides y el mismo himno nacional.

La cuarta área de conflicto es la de las confrontaciones lingüísticas (comprensión y lectoescritura). Estas se relacionan con el contenido de las materias y, en el caso estudiado, pasaron al plano personal de las interacciones diarias que tuvieron cuando llegaron a México. A pesar de este inconveniente, algunos de estos alumnos se valieron de diversas estrategias para traducir ciertos acontecimientos. Entre las estrategias generales que usaron siete de los nueve estudiantes retornados están el apoyo, dentro y fuera de clases, de las y los compañeros de escuela y de sus propios familiares, para resolver dudas sobre el 
significado de algunos conceptos después de haberlos consultado en internet (búsqueda y traducción). Estas estrategias les ayudaron a comprender algunos conceptos, pero todas y todos coincidieron en que les gustaría que la escuela los apoyara.

El quinto aspecto que identificaron es que «las y los docentes no les enseñan y no les tienen paciencia». Estos jóvenes aseguraron recibir de sus docentes un trato distinto del que le dan al resto de la clase. Por ejemplo, al levantar la mano, los docentes no atendían sus dudas o bien les decían que le preguntaran a un compañero de clase. Uno de los casos más significativos es el de una joven que se sintió discriminada por una docente, quien se dirigió a la joven y le manifestó en clase que era una incompetente en su materia por no ir a la par con el resto del grupo. La joven comentó que después de esa intervención no volvió a preguntarle nada a la maestra. Estos acontecimientos demuestran la escasez de estrategias de algunos docentes para recibir, atender y educar en contextos en que hay alumnos migrantes.

El sexto aspecto que identificaron las jóvenes y los jóvenes retornados en las secundarias de Mexicali es la inasistencia de alumnos («pintearse» las clases). Estos jóvenes perciben que esta acción no tiene sentido; cuestionan la actitud de los compañeros que la practican y piensan que, si no desean entrar a clases, sería mejor que no fueran a la escuela. Además, consideraron la inasistencia de sus compañeros como una acción retadora contra las autoridades educativas. Sin embargo, no solo los estudiantes faltan a clases: los encuestados se quejaron de que algunos docentes inciden en esta misma práctica.

Esta última cuestión conduce a la séptima inconformidad de la población de jóvenes retornados: la inasistencia de docentes (horas libres, maestros sustitutos). Estos actores son conscientes de que las y los docentes pueden faltar un par de días o semanas; sin embargo, en los colegios a los que asisten no llega un docente sustituto para dar continuidad a las clases, como ocurre en los EUA. Algunos jóvenes comentaron que, a un mes de empezar el ciclo escolar 2015-2016 no tenían profesores de remplazo en algunas materias. Una situación paralela es el caso de las «horas libres»: perciben estas licencias de forma negativa, pues consideran que es tiempo perdido durante el cual no aprenden nada.

El octavo conflicto sociocultural es que las y los docentes dejan muchas tareas para después de clases. Si la escuela es difícil para ellos, las tareas para hacer en casa lo son aún más. Así, estas actividades se vuelven un problema para el desarrollo educativo de dicha población, porque la mayoría de estos estudiantes no tienen el apoyo de sus padres, ya sea por su condición laboral o debido a que sus estudios son mínimos (secundaria o preparatoria). 
Continuando con las disonancias culturales, el noveno conflicto corresponde a las formas de evaluación (exámenes difíciles). Las y los jóvenes retornados no comprenden los criterios «subjetivos» con que algunos docentes evalúan el desempeño escolar, puesto que en ocasiones sobresale el «preferitismo» por algunos compañeros de clase. Estos estudiantes aseguraron estar preocupados en los exámenes finales porque no dominaban el idioma español. Cabe señalar que a veces reprobaban los exámenes por escribir mal o confundir palabras o enunciados. En opinión de estos alumnos, en México la educación no es incluyente en el momento de evaluar.

Franco (2017) afirma que la evaluación «tiene un papel sustantivo como indicador en los resultados del quehacer educativo nacional. De ser un instrumento interno de cada centro educativo con una función formativa, pasa a ser instrumento de medición de logros educativos» (pp. 707-708). Esta acción tiende a clasificar a las y los estudiantes y a develar el carácter discriminatorio del proceso de enseñanza-aprendizaje, por no contemplar los saberes que poseen los estudiantes retornados.

A manera de resumen, en la tabla 2 se muestra la forma en la que quedan representadas las disonancias culturales que vive la población de jóvenes retornados de los colegios de secundaria donde se adelantó el estudio.

\section{Tabla 2}

\section{Disonancias culturales jóvenes retornados en colegios de secundaria de Mexicali}

\begin{tabular}{ll} 
& - Las escuelas de México son de menor tamaño que las de los EUA. \\
Limitaciones del & - Áreas recreativas en mal estado (canchas de fútbol y de básquetbol). \\
espacio educativo & - Bibliotecas pequeñas. \\
& - Mobiliario en mal estado (pupitres, pizarrón, mesas y sillas de los docentes). \\
& - No existen casilleros. \\
\cline { 2 - 3 } Discontinuidad escolar/ & - Movilidad de un sistema educativo a otro (o de escuela). \\
sistema educativo & - Materias nuevas (Formación Cívica y Ética, e Historia de México). \\
& - Contenido de las materias. \\
& - Interacciones escolares entre alumnos (proxemia). \\
& - «Carilla», bullying o «cura» (falta de respeto de los alumnos). \\
& - Acto cívico (honores a la bandera con duración de una hora o más). \\
& - Confrontaciones lingüísticas (comprensión y lectoescritura). \\
& - Los maestros no enseñan y no tienen paciencia. \\
& - Inasistencia de estudiantes («pintearse» las clases) y de docentes (horas libres). \\
& - Dejan muchas tareas. \\
& - Exámenes difíciles (formas de evaluación).
\end{tabular}


Si bien las disonancias culturales que viven las jóvenes y los jóvenes retornados en colegios de secundaria de Mexicali son evidentes, hay que determinar la desinformación de la comunidad educativa sobre la población migrante. En general, se observa que las y los estudiantes retornados son invisibilizados por la comunidad educativa en las áreas de identificación, atención y seguimiento.

\section{Discusión}

Al llegar a México, las y los jóvenes retornados cambian de un sistema educativo a otro (o de escuela), y en ese proceso deben adecuarse a los cánones de los centros educativos mexicanos. El conflicto que identifica esta población al pasar de un sistema a otro se centra en la segunda disonancia cultural: discontinuidad escolar/sistema educativo. De acuerdo con Franco (2017), la escuela actúa como normalizadora de los sujetos que no se ajustan a los cánones sociales, sobre todo por diferencias de clase, étnicas y de condición etaria. Así, estos estudiantes que retornan viven una realidad trastocada en sus relaciones sociales y escolares, lo cual, en ciertos casos, implica volver a empezar un ciclo escolar en otro país, retomar rutinas, aprender y convivir con el otro.

Las disonancias culturales por las que atraviesa la población de jóvenes retornados tienen que ver con los usos y costumbres del sistema escolar mexicano y todas sus implicaciones. Esos aspectos están estrechamente vinculados con las limitaciones que perciben los actores, relacionadas con la infraestructura escolar y sus condiciones: el ambiente, el equipamiento y el mobiliario escolar, así como los materiales educativos de los que se valen las y los docentes en sus clases, todo lo cual forma parte de la realidad de la escolaridad. De ahí que se analice el proceso de incorporación o reincorporación sociocultural que experimentan los alumnos migrantes. Además de las disonancias culturales, Vargas y Camacho (2019) identificaron situaciones de desigualdad y discriminación contra la población migrante en escuelas de Tijuana, que los impulsaban a abandonar sus estudios y a esperar la mayoría de edad para regresar a los EUA a trabajar.

De acuerdo con lo expuesto, es necesario estudiar este fenómeno desde otros enfoques para conocer aristas aún no contempladas. Así, los estudios interculturales podrían aportar el «modelo cultural interno» (Charle, et al., 2006) presente en las y los jóvenes retornados. Dietz y Mateos (2013) afirman que el modelo cultural interno «da elementos para explicar la forma en que las personas piensan y actúan [...]; está integrado por aquellas 
reflexiones que [los] actores establecen como punto de referencia para explicarse, definir, reproducir la interculturalidad» (p. 46). Así, este modelo ofrece la pauta para conocer al otro y construir un espacio para todos.

Por su parte, Franco (2017) menciona que, a pesar de las construcciones históricas de los sistemas educativos de México y los EUA, ambos asimilan al diferente o lo excluyen cuando este se resiste a adaptarse al contexto donde se encuentra. Esto hace que las y los estudiantes retornados difícilmente tengan éxito en sus estudios. Esta parte debe repensarse para que la población migrante en edad escolar obligatoria se deconstruya y el Estado replantee políticas educativas que tomen en cuenta sus narrativas con el objetivo de crear un currículo que se adecúe a las exigencias contemporáneas. De esta forma, la inclusión (una que atienda las diferentes necesidades de los grupos emergentes) es el principal aspecto que debe tenerse en cuenta a la hora de construir una sociedad diversa.

Las nuevas generaciones de jóvenes retornados experimentan dinámicas que no se han contemplado. En consecuencia, es necesario adoptar una postura crítica y debatir si será posible la inclusión en México a partir del reordenamiento social que experimenta la sociedad mexicana, y si la educación es o será de excelencia. Así, un reto para estas investigaciones consistiría en usar métodos biográficos que, en las escuelas, podrían implementarse en el área de psicopedagogía o en la de orientación educativa. De esta manera, las vivencias y experiencias de la población migrante, expresadas mediante narrativas, servirían para construir un nuevo panorama social y cultural en el país.

Esta problemática también alerta sobre cómo dicha población va forjando y transformando su identidad en las escuelas, y transciende sus muros. En cuanto al sistema educativo mexicano, le falta abordar la inclusión escolar de la población migrante mestiza. Le compete al Gobierno nacional atender este problema, que debe tratarse desde las escuelas, las políticas públicas, la Secretaría de Educación Pública, la Secretaría de Educación y Bienestar Social y las universidades, en vista de las crecientes oleadas de inmigrantes centroamericanos y las políticas antiinmigrantes del Gobierno estadounidense.

\section{Agradecimientos}

Se extiende un agradecimiento al Consejo Nacional de Ciencia y Tecnología del Gobierno de México por financiar este proyecto, y a las jóvenes y los jóvenes retornados e (in)migrantes que colaboraron con la investigación. 


\section{Referencias}

Aguilar, R., \& Ortiz, L. C. (2018). La emergente generación 1.5.: estadounidenses creciendo en México. En C. A. Garrido \& J. Anderson (coords.), ¿Santuarios educativos en México? Proyectos y propuestas ante la criminalización de jóvenes dreamers, retornados y deportados (pp. 127-150). Universidad Veracruzana. https://doi.org/10.25009/ uv.2020.655

Blanco, C. (2011). Encuesta y estadística: métodos de investigación cuantitativa en ciencias sociales y comunicación. Brujas.

Bustamante, P. del R. (2016). Disonancias culturales entre alumnos retornados de EUA a Mexicali, Baja California [Tesis de maestría]. Instituto de Investigaciones Culturales; Museo UABC.

Charle, C., Schriewer, J., \& Wagner, P. (comps.) (2006). Redes intelectuales transnacionales: formas de conocimiento académico y búsqueda de identidades culturales. Pomares.

Coll, C. (1984). Estructura grupal, interacción entre alumnos y aprendizaje escolar. Infancia y aprendizaje. Journal for the Study of Education and Development, 27-28, 119-138. https://doi.org/10.1080/02103702.1984.10822047

Comisión Nacional de Derechos Humanos México (CNDH) (2018). Derechos de niñas, ninos y adolescentes migrantes y refugiados: una guía para su protección. CNDH.

Deal, T. E., \& Peterson, K. D. (2009). Shaping school culture: Pitfalls, paradoxes, \& promises. Josey-Bass.

Dietz, G., \& Mateos, S. (2013). Interculturalidad y educación intercultural en México: un análisis de los discursos nacionales e internacionales en su impacto en los modelos educativos mexicanos. Secretaría de Educación Pública.

Dirección General de Relaciones Internacionales (DGRI) (2015). Condiciones contemporáneas de la relación México-Estados Unidos: hacia una movilidad estudiantil y académica. https://www.gob.mx/cms/uploads/attachment/file/133283/nazzar.pdf

Franco, M. (2017). Sistemas educativos y migración: una mirada a la educación en Estados Unidos y México. Revista Mexicana de Investigación Educativa, 22(74), 705-728.

García, I., \& Burgueño, N. (2017). Menores transnacionales: el proceso de incorporación sociocultural en el espacio educativo. El caso de la comunidad de Cosalá, Sinaloa. En G. C. Valdez \& I. García (coords.), Tránsito y retorno de la niñez migrante: epílogo en la administración de Trump. El Colegio de Sonora; Universidad de Sinaloa.

Glaser, B. G. (1992). Basics of grounded theory analysis. Sociology Press. 
Goffman, E. (2001). Internados: ensayos sobre la situación social de los enfermos mentales. Amorrortu.

Instituto de los Mexicanos en el Exterior (IME) (2016). Programa Binacional de Educación Migrante. https://www.gob.mx/ime/acciones-y-programas/programa-binacional-deeducacion-migrante-probem-61464

Instituto Nacional para la Evaluación de la Educación (INEE) (2018). La educación obligatoria en México: Informe 2018. https://www.inee.edu.mx/wp-content/uploads/ 2018/12/P1I243.pdf

Jacobo-Suárez, M. (2017). De regreso a «casa» y sin apostilla: estudiantes mexicoamericanos en México. Sinéctica, 18, 1-18.

Martínez-Otero, V. (2007). La buena educación: reflexiones y propuestas de psicopedagogía humanista. Anthropos.

Nateras, A. (2010). Adscripciones identitarias juveniles: tiempo y espacio social. El cotidiano, 173, 17-23.

Programa Binacional de Educación Migrante (Probem) (2018). Estadísticas de estudiantes extranjeros en Mexicali. Probem.

Rockwell, E. (coord.) (1995). La escuela cotidiana. Fondo de Cultura Económica.

Rockwell, E. (2015). La experiencia etnográfica: historia y cultura en los procesos educativos. Paidós.

Romo, V. (2012). Espacios educativos desafiantes en educación infantil. En V. Peralta \& L. Hernández (coords.), Antología de experiencias de la educación inicial iberoamericana (pp. 141-145). OEI y Unicef.

Taylor, S. J., \& Bogdan, R. (1990). Introducción a los métodos cualitativos de investigación. Paidós.

Terigi, F. Z. (2010). Las cronologías de aprendizaje: un concepto para pensar las trayectorias escolares. Ministerio de Cultura y Educación.

Valdez, G. C., \& García, I. (coords.) (2017). Tránsito y retorno de la niñez migrante: epílogo en la administración de Trump. El Colegio de Sonora; Universidad de Sinaloa.

Valdez, G. C., \& Ruiz, L. F. (2017). Sonora en la migración internacional: menores migrantes de retorno. Entre la reforma educativa y los procesos institucionales estatales. En G. Valdez \& I. García (coords.), Tránsito y retorno de la niñez migrante: epílogo en la administración de Trump (pp. 145-173). El Colegio de Sonora; Universidad de Sinaloa.

Vargas, E. D. (2019). Los desafíos para la inclusión educativa de los migrantes de Estados Unidos a México. En E. Corzo (coord.), Migración de retorno y derechos sociales: barreras a la 
integración (pp. 21-3o). https://migracionderetorno.colmex.mx/wp-content/uploads/ 2019/06/COMPILADO_WEB.pdf

Vargas, E. D., \& Camacho, E. (2019). Desigualdad e inserción escolar de niños inmigrantes de Estados Unidos a Baja California. En M. París, A. Hualde \& O. Woo (coords.), Experiencias de retorno de migrantes mexicanos en contextos urbanos (pp. 227-259). Colegio de la Frontera Norte.

Velasco, H., \& Díaz de Rada, A. (1997). La lógica de la investigación etnográfica: un modelo de trabajo para etnógrafos de la escuela. Trotta.

Zúñiga, V. (2017). Los niños y las niñas migrantes en escena. Sinéctica, 48, 1-20. https:// sinectica.iteso.mx/index.php/sinectica/article/view/70o/712 